\title{
New insights in low-energy electron-fullerene interactions
}

\author{
Alfred Msezane ${ }^{\dagger 1}$, Zineb Felfli $^{\dagger 2}$ \\ ${ }^{\dagger}$ Department of Physics and CTSPS, Clark Atlanta University, Atlanta, Georgia 30314, USA
}

\begin{abstract}
Synopsis Regge pole calculated low-energy electron scattering total cross sections (TCSs) for ground and first excited states of fullerenes are generally robust; for $\mathrm{C}_{20}$ they resemble those of atomic Au. Here we explore the variation of $\mathrm{R}$, the ratio of second to first Ramsauer-Townsend minima of excited states TCSs, from $C_{20}$ through $C_{70}$. We find that for $C_{20} R$ is close to that for $A u\left(>1\right.$.) while for $C_{24} R$ is about unity. For $\mathrm{C}_{70} \mathrm{R}$ is less than 0.5 demonstrating strong departure from atomic behavior due to significant polarization interaction in $\mathrm{C}_{70}$; which also induces long-lived metastable anions in $\mathrm{C}_{70}$.
\end{abstract}

The discovery of the now most studied $\mathrm{C}_{60}$ fullerene [1] inspired vigorous searches for new fullerenes, leading to the discovery of the smallest fullerene thought to exist, $\mathrm{C}_{20}$. Its electron affinity (EA) was measured to be $2.25 \mathrm{eV}$ [2]. Other small fullerenes of interest here are $\mathrm{C}_{24}, \mathrm{C}_{26}, \mathrm{C}_{28}$ and $\mathrm{C}_{44}$, some of which have measured EAs available.

This investigation has been motivated by: 1) Amusia concluded that fullerenes can be viewed as "big atoms" [3]; 2) Rich resonances have been observed to characterize low-energy electron-fullerene interactions; 3) In [4] the Regge pole calculated low-energy electron elastic total cross sections (TCSs) for ground states $\mathrm{C}_{60}$ through $\mathrm{C}_{92}$ were found to be characterized by dramatically sharp resonances located at the second Ramsauer-Townsend (R-T) minima of their TCSs. The energy positions of these sharp resonances were generally in outstanding agreement with the measured EAs for the investigated fullerenes and 4) The calculated TCSs revealed polarization induced long-lived metastable fullerene anions [4].

The departure from atomic behavior is studied through the ratio $\mathrm{R}$ focusing on the green curve of TCSs for $\mathrm{C}_{20}$ through $\mathrm{C}_{70}$. In the Fig. the first R-T minima for both $\mathrm{C}_{20}$ and $\mathrm{Au}$ are lower than the second, indicative of atomic behavior. For $\mathrm{C}_{26}, \mathrm{R}$ is about unity and for $\mathrm{C}_{70}$ it is < 0.5, demonstrating complete departure and conversion of the $0.466 \mathrm{eV}$ resonance to a longlived metastable anion in $\mathrm{C}_{70}$.

\footnotetext{
${ }^{1}$ E-mail: amsezane@ cau.edu

${ }^{2}$ E-mail: zfelfli@cau.edu
}

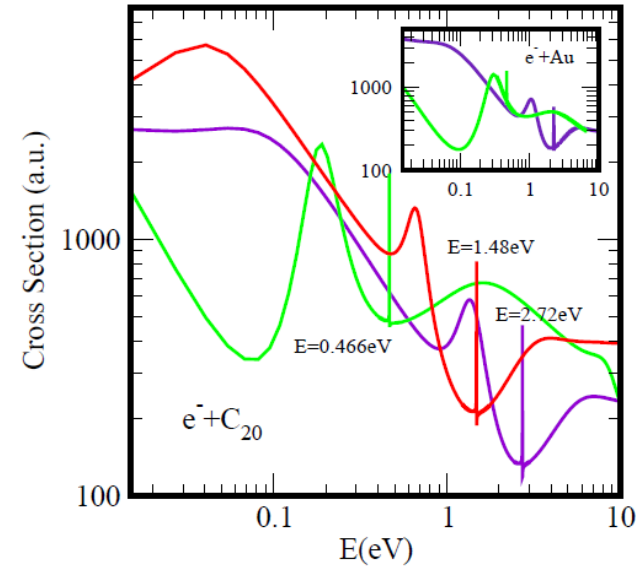

Figure 1: Total cross sections (a.u.) for electron elastic scattering versus $\mathrm{E}(\mathrm{eV})$ for $\mathrm{C}_{20}$. The purple, pink and green curves represent results for the ground, induced metastable and first excited states, respectively; the insert represents TCSs for atomic Au. The dramatically sharp resonances correspond to fullerene anions formation during the collisions.

Research is supported by U.S. DOE, OBES, OS

\section{References}

[1] H. W. Kroto et al. 1985 Nature $\mathbf{3 1 8} 162$

[2] H. Prinzbach et al. 2000 Nature $\mathbf{4 0 7} 60$

[3] M. Ya Amusia 2013 Chem Phys $\underline{\mathbf{4 1 4}} 168$

[4] Z. Felfli and A. Z. Msezane 2017 J. Phys. B (Submitted). 